Proceedings of the 2011 Winter Simulation Conference

S. Jain, R. R. Creasey, J. Himmelspach, K. P. White, and M. Fu, eds.

\title{
DISCRETE OPTIMIZATION VIA APPROXIMATE ANNEALING ADAPTIVE SEARCH WITH STOCHASTIC AVERAGING
}

\author{
Jiaqiao $\mathrm{Hu}$ \\ Chen Wang \\ Department of Applied Mathematics and Statistics \\ State University of New York at Stony Brook, \\ Stony Brook, NY 11794, USA
}

\begin{abstract}
We propose a random search algorithm for black-box optimization with discrete decision variables. The algorithm is based on the recently introduced Model-based Annealing Random Search (MARS) for global optimization, which samples candidate solutions from a sequence of iteratively focusing distribution functions over the solution space. In contrast with MARS, which requires a sample size (number of candidate solutions) that grows at least polynomially with the number of iterations for convergence, our approach employs a stochastic averaging idea and uses only a small constant number of candidate solutions per iteration. We establish global convergence of the proposed algorithm and provide numerical examples to illustrate its performance.
\end{abstract}

\section{INTRODUCTION}

Many systems arising in engineering design, biostatistics, and manufacturing are characterized by complex nonlinearities in their dynamics, and frequently require black-box evaluations to assess their performances, which are unlikely to exhibit any nice structural properties. The need for optimization of such systems has led to the research over the past few decades focusing on developing random search techniques that rely only on the system performance measures in finding improved candidate solutions. Examples of these techniques are simulated annealing (Kirkpatrick, Gelatt, and Vecchi 1983), genetic algorithms (Goldberg 1989), tabu search (Glover 1990), nested partitions (Shi and Ólafsson 2000), adaptive random search (Zabinksy 2003), and a class of algorithms collectively known as the model-based methods, which include ant colony optimization (Dorigo and Gambardella 1997), estimation of distribution algorithms (Larranaga and Lozano 2002), the cross-entropy method (Rubinstein and Kroese 2004), and model reference adaptive search (MRAS) (Hu, Fu, and Marcus 2007).

Among the class of model-based algorithms, the Annealing Adaptive Search (AAS) (Romeijn and Smith 1994, Zabinksy 2003) has been well studied in literature and found to have the prominent property that its computational complexity increases at most linearly with the problem dimension for a large class of optimization problems (e.g., Zabinksy 2003). However, to successfully implement AAS in practice, one is required to generate samples/candidate solutions exactly from a sequence of iteratively focusing Boltzmann distributions parameterized by time-dependent temperatures, which is known to be extremely difficult. This implementation difficulty was previously addressed primarily through sampling techniques based on Markov-chain Monte Carlo. Recently, $\mathrm{Hu}$ and $\mathrm{Hu}$ (2010) proposed an alternative approach called Model-based Annealing Random Search (MARS) to overcome this difficulty, where the underlying idea is to construct a sequence of surrogate distributions to successively approximate the target Boltzmann distributions and then use the surrogate distributions to generate candidate solutions. By connecting MARS to recursions of stochastic approximation (SA) type (Evans and Weber 1986, Kushner and Yin 1997, Robbins and Monro 1951, Spall 2003), it has been shown in $\mathrm{Hu}$ and $\mathrm{Hu}$ (2010) that the algorithm converges 


\section{$H u$ and Wang}

to the global optimal solution at the expense of using a per-iteration computational effort that increases polynomially with the number of iterations.

However, it is well-known that SA algorithms may exhibit slow convergence behavior. Thus, using a sample size that increases polynomially in MARS could lead to a computational burden that becomes prohibitive as the search proceeds. Furthermore, as in a typical model-based algorithm, a whole new set of solutions needs to be generated and evaluated at each iteration, and all the solutions sampled during the previous iterations are discarded. In this paper, we aim to improve the computational efficiency of MARS on optimization problems over discrete-valued domains. In particular, we propose an algorithm that combines MARS with an additional stochastic averaging procedure, so that at each iteration of the new algorithm, all candidate solutions generated in the previous iterations contribute to the construction of the current surrogate distribution. As a result, the number of samples per iteration can be significantly reduced or be held at a small constant value. Our preliminary empirical results indicate that the new algorithm can be more efficient (in term of the number of performance evaluations) than the original MARS algorithm.

The rest of the paper is organized as follows. In Section 2, we introduce the problem setting and describe the proposed algorithm. In Section 3, we prove the global convergence of the algorithm, followed by preliminary computational experiments in Section 4 to illustrate its performance. Finally, we conclude the paper in Section 5.

\section{MARS WITH STOCHASTIC AVERAGING}

We consider the following general discrete optimization problem:

$$
x^{*} \in \underset{x \in \mathbb{X}}{\arg \max } H(x),
$$

where $x:=\left(x_{1}, \ldots, x_{n}\right)^{T}$ is the vector of $n$ decision variables, the solution space $\mathbb{X} \subset \Re^{n}$ is a (finite) discrete set, and $H: X \rightarrow \Re$ is a deterministic bounded objective function. Throughout this paper, we assume there exists a unique optimal solution $x^{*}$ to (1). Without loss of generality, we also assume that $H(x) \geq 0 \forall x \in \mathbb{X}$ and each component of $x, x_{i}$, takes values from a set of $m$ distinct values $\left\{a_{i}^{1}, \ldots, a_{i}^{m}\right\}$. Thus, the size of the solution space $|\mathbb{X}|=m^{n}$.

In an attempt at solving (1), the idealized AAS assumes that candidate solutions can be generated exactly, at each iteration $k$, from the Boltzmann distribution

$$
g_{k}(x)=\frac{e^{H(x) / T_{k}}}{\sum_{x \in \mathbb{X}} e^{H(x) / T_{k}}} \forall x \in \mathbb{X},
$$

where $T_{k}$ is an iteration-dependent temperature parameter. It can be shown that as $T_{k}$ decreases to zero, the Boltzmann distribution $g_{k}$ will converge to a limiting distribution $g^{*}$ assigning unit mass to the optimal $x^{*}$. Thus, in the long run, the AAS algorithm ensures that the optimal solution will be sampled with probability one. However, in practice, the sequence $\left\{g_{k}\right\}$ is unknown a priori unless the entire solution space can be explicitly enumerated. In the recently proposed MARS algorithm, this implementation difficulty is circumvented by sampling solutions from a parameterized surrogate distribution that approximates $g_{k}$. Specifically, within the context of problem (1), the parameterized distribution can be specified in terms of an $n$-by- $m$ stochastic matrix $q$ with its $(i, j)$ th entry $q(i, j) \in[0,1]$ representing the probability that the $i$ th decision variable $x_{i}$ takes the $j$ th value $a_{i}^{j}$. Such a stochastic matrix $q$ induces a probability mass function (p.m.f.) over $\mathbb{X}$ where $q$ itself can be viewed as the parameter of the distribution:

$$
\phi(x, q):=\prod_{i=1}^{n} \prod_{j=1}^{m}[q(i, j)]^{I\left\{x \in \Lambda_{i, j}\right\}} \quad \forall x \in \mathbb{X},
$$

where $I\{\cdot\}$ is the indicator function and $\Lambda_{i, j}:=\left\{x \in \mathbb{X}: x_{i}=a_{i}^{j}\right\}$ is the collection of feasible solutions

whose $i$ th components assume the value $a_{i}^{j}$. The idea now is to find the parameter $q_{k}$ of the p.m.f. $\phi$ so that 


\section{Hu and Wang}

$g_{k}$ can be closely approximated by $\phi\left(\cdot, q_{k}\right)$. A common implementation minimizes the Kullback-Leibler (KL) divergence between $g_{k}$ and $\phi(\cdot, q)$ (e.g., Rubinstein and Kroese 2004, Wolpert 2004), i.e.,

$$
q_{k}=\underset{\sum_{j} q(i, j)=1 \forall i}{\arg \min } \mathscr{D}\left(g_{k} \mid \phi(\cdot, q)\right):=\underset{\sum_{j} q(i, j)=1 \forall i}{\arg \min } E_{g_{k}}\left[\ln \frac{g_{k}(X)}{\phi(X, q)}\right]
$$

where $E_{g_{k}}[\cdot]$ is the expectation with respect to $g_{k}$ and $X \sim g_{k}$ is a random vector taking values from $\mathbb{X}$. By dropping terms that are constant with respect to $q$, Equation (3) can be written as

$$
q_{k}=\underset{\sum_{j} q(i, j)=1 \forall i}{\arg \max }\left\{Q_{k}(q):=\sum_{x \in \mathbb{X}} e^{H(x) / T_{k}} \ln \phi(x, q)\right\} .
$$

In practical implementation of MARS, the above $Q$-function is estimated by first generating $N$ i.i.d. candidate solutions $X_{k-1}^{1}, \ldots, X_{k-1}^{N}$ from $\phi\left(x, q_{k-1}\right)$ (i.e., the p.m.f. parameterized by the $q$ matrix obtained at iteration $k-1)$, and then replacing $Q_{k}(q)$ by its sample average approximation

$$
\bar{Q}_{k}(q):=\frac{1}{N} \sum_{\ell=1}^{N} e^{H\left(X_{k-1}^{\ell}\right) / T_{k}} \phi^{-1}\left(X_{k-1}^{\ell}, q_{k-1}\right) \ln \phi\left(X_{k-1}^{\ell}, q\right) .
$$

Although $\bar{Q}_{k}(q)$ provides an unbiased estimate of $Q_{k}(q)$, the corresponding optimization step will lead to an estimate of $q_{k}$ that is biased for any finite sample size $N$, because the optimal solution to (4) involves a ratio of sums/expectations (due to the logarithm function). Consequently, the conditions for convergence of MARS (Hu and $\mathrm{Hu} 2010$ ) (as well as other algorithms like CE and MRAS; cf. e.g., Hu, Fu, and Marcus 2007, $\mathrm{Hu}$ and $\mathrm{Hu} 2009$ ), require the use of a sample size $N$ that increases at least polynomially with $k$ in order to reduce the ratio bias effect.

In this paper, we examine an alternative approach to address this bias issue, where the basic idea is to replace the sample average $\bar{Q}_{k}(q)$ with a stochastic averaging procedure in estimating the Q-function, i.e.,

$$
\hat{Q}_{k}(q)=\left(1-\alpha_{k-1}\right) \hat{Q}_{k-1}(q)+\alpha_{k-1} \frac{1}{N} \sum_{\ell=1}^{N} e^{H\left(X_{k-1}^{\ell}\right) / T_{k}} \phi^{-1}\left(X_{k-1}^{\ell}, q_{k-1}\right) \ln \phi\left(X_{k-1}^{\ell}, q\right),
$$

where $\hat{Q}_{1}(q)=\frac{1}{N} \sum_{\ell=1}^{N} e^{H\left(X_{0}^{\ell}\right) / T_{1}} \phi^{-1}\left(X_{0}^{\ell}, q_{0}\right) \ln \phi\left(X_{0}^{\ell}, q\right)$ with $X_{0}^{1} \ldots, X_{0}^{N}$ i.i.d. $\sim \phi\left(x, q_{0}\right)$, and $\left\{\alpha_{k}\right\}$ is a step size/gain sequence with $\alpha_{k} \in(0,1] \forall k$. Note that the above procedure makes a more efficient use of the past sampling information. In particular, due to the recursive nature of (5), all candidate solutions generated in the previous iterations contribute to the estimation of the $Q$-function $Q_{k}(q)$. As a result, it is intuitively clear that the number of samples per iteration $N$ can be significantly reduced or even be held at a small constant value.

Since the above recursion updates an entire function of $q$, direct implementation of (5) can be difficult. However, by the definition of the parameterized p.m.f. $\phi$ (cf. (2)), it is easy to observe that when $k=2$,

$$
\hat{Q}_{2}(q)=\sum_{i=1}^{n} \sum_{j=1}^{m} \ln q(i, j)\left[\frac{1-\alpha_{1}}{N} \sum_{\ell=1}^{N} e^{\frac{H\left(X_{0}^{\ell}\right)}{T_{1}}} \phi^{-1}\left(X_{0}^{\ell}, q_{0}\right) I\left\{X_{0}^{\ell} \in \Lambda_{i, j}\right\}+\frac{\alpha_{1}}{N} \sum_{\ell=1}^{N} e^{\frac{H\left(X_{1}^{\ell}\right)}{T_{2}}} \phi^{-1}\left(X_{1}^{\ell}, q_{1}\right) I\left\{X_{1}^{\ell} \in \Lambda_{i, j}\right\}\right] .
$$

Furthermore, an inductive argument shows that $\hat{Q}_{k}(q)$ can be expressed recursively as

$$
\hat{Q}_{k}(q)=\sum_{i=1}^{n} \sum_{j=1}^{m} \ln q(i, j) S_{k}(i, j),
$$


where

$$
\begin{aligned}
& S_{k}(i, j)=S_{k-1}(i, j)+\alpha_{k-1}\left[\frac{1}{N} \sum_{\ell=1}^{N} e^{H\left(X_{k-1}^{\ell}\right) / T_{k}} \phi^{-1}\left(X_{k-1}^{\ell}, q_{k-1}\right) I\left\{X_{k-1}^{\ell} \in \Lambda_{i, j}\right\}-S_{k-1}(i, j)\right] \text { and } \\
& S_{1}(i, j)=\frac{1}{N} \sum_{\ell=1}^{N} e^{H\left(X_{0}^{\ell}\right) / T_{1}} \phi^{-1}\left(X_{0}^{\ell}, q_{0}\right) I\left\{X_{0}^{\ell} \in \Lambda_{i, j}\right\} .
\end{aligned}
$$

Thus, by substituting $\hat{Q}_{k}(q)$ for $Q_{k}(q)$ in (4), we have the following optimization problem:

$$
\begin{aligned}
& \hat{q}_{k}=\arg \max \sum_{i=1}^{n} \sum_{j=1}^{m} \ln q(i, j) S_{k}(i, j) \\
& \text { s.t. } \sum_{j=1}^{m} q(i, j)=1 \text { and } q(i, j) \geq 0 \forall i=1, \ldots, n, j=1, \ldots, m .
\end{aligned}
$$

The problem can be conveniently solved by a simple application of the Lagrange multiplier theorem, yielding a closed-form expression for each entry of the $\hat{q}_{k}$ matrix

$$
\hat{q}_{k}(i, j)=\frac{S_{k}(i, j)}{\sum_{j=1}^{m} S_{k}(i, j)} \forall i, j .
$$

This leads to the proposed algorithm we call MARS with stochastic averaging (MARS-SA):

\section{MARS with Stochastic Averaging (MARS-SA)}

Step 0: Set $\hat{q}_{0}(i, j)=\frac{1}{m} \forall i, j$. Specify an annealing schedule $\left\{T_{k}\right\}$, a step-size sequence $\left\{\alpha_{k}\right\}$, a sample size $N$, and a parameter $\beta \in(0,1)$. Set iteration counter $k=0$.

Step 1: Independently generate $N$ candidate solutions $X_{k}^{1}, \ldots, X_{k}^{N}$ from the p.m.f. $\hat{\phi}\left(x, \hat{q}_{k}\right)=(1-\beta) \phi\left(x, \hat{q}_{k}\right)+\beta \phi\left(x, \hat{q}_{0}\right)$.

Step 2: If $k=0$ set

$$
S_{1}(i, j)=\frac{1}{N} \sum_{\ell=1}^{N} e^{H\left(X_{0}^{\ell}\right) / T_{1}} \phi^{-1}\left(X_{0}^{\ell}, \hat{q}_{0}\right) I\left\{X_{0}^{\ell} \in \Lambda_{i, j}\right\} ;
$$

otherwise update $S_{k+1}$ as

$$
S_{k+1}(i, j)=S_{k}(i, j)+\alpha_{k}\left[\frac{1}{N} \sum_{\ell=1}^{N} e^{H\left(X_{k}^{\ell}\right) / T_{k+1}} \hat{\phi}^{-1}\left(X_{k}^{\ell}, \hat{q}_{k}\right) I\left\{X_{k}^{\ell} \in \Lambda_{i, j}\right\}-S_{k}(i, j)\right] .
$$

Step 3: Compute $\hat{q}_{k+1}$ according to (6).

Step 4: If a stopping rule is satisfied, then terminate; otherwise set $k=k+1$ and go to Step 1 .

Note that at Step 1 of MARS-SA, instead of directly sampling solutions from the current parameterized distribution $\phi\left(x, \hat{q}_{k}\right)$, we have used a p.m.f. $\hat{\phi}\left(x, \hat{q}_{k}\right)$, which is the mixture of $\phi\left(x, \hat{q}_{k}\right)$ with the initial (discrete uniform) p.m.f. $\phi\left(x, \hat{q}_{0}\right)$. This allows the algorithm to explore the entire solution space, so that every candidate solution in $\mathbb{X}$ will be sampled at least with probability $\beta(1 / m)^{n}$ at each single iteration.

\section{GLOBAL CONVERGENCE OF MARS-SA}

We start by defining some notations. Let $P(\cdot)$ and $E[\cdot]$ be the probability and expectation taken with respect to the random selection of candidate solutions at Step 1 of the algorithm. Throughout this paper, probability one (almost sure) convergence and boundedness shall be understood with respect to $P$. Define 


\section{Hu and Wang}

$\mathscr{F}_{k}=\sigma\left\{X_{0}^{\ell}, \ldots, X_{k-1}^{\ell}, \quad \ell=1, \ldots, N\right\}$ as the increasing $\sigma$-fields generated by the collection of random solutions obtained up to iteration $k-1$. Note that given $\mathscr{F}_{k}$, the parameter $\hat{q}_{k}$ is completely determined, and the conditional probability and expectation $P\left(\cdot \mid \mathscr{F}_{k}\right)$ and $E\left[\cdot \mid \mathscr{F}_{k}\right]$ are to be understood with respect to $\hat{\phi}\left(x, \hat{q}_{k}\right)$.

To analyze the MARS-SA algorithm, we rewrite recursion (7) in the following equivalent form

$$
S_{k+1}(i, j)=S_{k}(i, j)+\alpha_{k}\left[\sum_{x \in \mathbb{X}} e^{H(x) / T_{k+1}} I\left\{x \in \Lambda_{i, j}\right\}-S_{k}(i, j)\right]+\alpha_{k} \zeta_{k}(i, j),
$$

where $\zeta_{k}(i, j)=\frac{1}{N} \sum_{\ell=1}^{N} e^{H\left(X_{k}^{\ell}\right) / T_{k+1}} \hat{\phi}^{-1}\left(X_{k}^{\ell}, \hat{q}_{k}\right) I\left\{X_{k}^{\ell} \in \Lambda_{i, j}\right\}-\sum_{x \in \mathbb{X}} e^{H(x) / T_{k+1}} I\left\{x \in \Lambda_{i, j}\right\}$. We further define the scaled versions of $S_{k}$ and $\zeta_{k}$ as

$$
U_{k}(i, j)=\frac{S_{k}(i, j)}{\sum_{\mathbb{X}} e^{H(x) / T_{k}}} \text { and } \eta_{k}(i, j)=\frac{\zeta_{k}(i, j)}{\sum_{\mathbb{X}} e^{H(x) / T_{k+1}}} .
$$

The following is a well-known result, which states that the sequence of Boltzmann distributions $\left\{g_{k}\right\}$ converges to a degenerate distribution that concentrates only on the optimal solution $x^{*}$. We present its proof for completeness.

Lemma 1 If $T_{k} \rightarrow 0$ as $k \rightarrow \infty$, then $E_{g_{k}}\left[I\left\{X \in \Lambda_{i, j}\right\}\right] \rightarrow I\left\{x^{*} \in \Lambda_{i, j}\right\}$ as $k \rightarrow \infty$.

Proof.

$$
\begin{aligned}
\left|E_{g_{k}}\left[I\left\{X \in \Lambda_{i, j}\right\}\right]-I\left\{x^{*} \in \Lambda_{i, j}\right\}\right| & \leq E_{g_{k}}\left[\left|I\left\{X \in \Lambda_{i, j}\right\}-I\left\{x^{*} \in \Lambda_{i, j}\right\}\right|\right] \\
& =\sum_{x \neq x^{*}}\left|I\left\{X \in \Lambda_{i, j}\right\}-I\left\{x^{*} \in \Lambda_{i, j}\right\}\right| \frac{e^{H(x) / T_{k}}}{\sum_{\mathbb{X}} e^{H(x) / T_{k}}} \\
& \leq \frac{\sum_{x \neq x^{*}} e^{\left(H(x)-H\left(x^{*}\right)\right) / T_{k}}}{1+\sum_{x \neq x^{*}} e^{\left(H(x)-H\left(x^{*}\right)\right) / T_{k}}} \\
& \leq \sum_{x \neq x^{*}} e^{\left(H(x)-H\left(x^{*}\right)\right) / T_{k}}
\end{aligned}
$$

which vanishes to zero as $T_{k} \rightarrow 0$, since $H(x)<H\left(x^{*}\right) \forall x \neq x^{*}$.

The next lemma shows that $U_{k}$ is bounded w.p.1.

Lemma 2 Assume $T_{k} \geq T_{k+1}>0$ for all $k$. Then $U_{k}(i, j)$ is bounded w.p.1. $\forall i, j$.

Proof. Dividing both sides of (8) by $\sum_{\mathbb{X}} e^{H(x) / T_{k+1}}$, we obtain

$$
\begin{aligned}
U_{k+1}(i, j) & =\left(1-\alpha_{k}\right) \frac{S_{k}(i, j)}{\sum_{\mathbb{X}} e^{H(x) / T_{k+1}}}+\alpha_{k} \frac{\sum_{x \in \mathbb{X}} e^{H(x) / T_{k+1}} I\left\{x \in \Lambda_{i, j}\right\}}{\sum_{\mathbb{X}} e^{H(x) / T_{k+1}}}+\alpha_{k} \eta_{k}(i, j) \\
& \leq\left(1-\alpha_{k}\right) U_{k}(i, j)+\alpha_{k}+\alpha_{k} \eta_{k}(i, j) \quad \text { since } T_{k} \geq T_{k+1}>0 .
\end{aligned}
$$

This implies inductively that

$$
\begin{aligned}
U_{k+1}(i, j) & \leq \prod_{r=1}^{k}\left(1-\alpha_{r}\right) U_{1}(i, j)+\sum_{r=1}^{k}\left[\prod_{s=r+1}^{k}\left(1-\alpha_{s}\right)\right] \alpha_{r}+\sum_{r=1}^{k}\left[\prod_{s=r+1}^{k}\left(1-\alpha_{s}\right)\right] \alpha_{r} \eta_{r}(i, j) \\
& \leq U_{1}(i, j)+1+Y_{k}(i, j),
\end{aligned}
$$




\section{Hu and Wang}

where $Y_{k}(i, j)=\sum_{r=1}^{k}\left[\prod_{s=r+1}^{k}\left(1-\alpha_{s}\right)\right] \alpha_{r} \eta_{r}(i, j)$. Here we have used the following fact:

$$
\begin{aligned}
\sum_{r=1}^{k}\left[\prod_{s=r+1}^{k}\left(1-\alpha_{s}\right)\right] \alpha_{r} & =\sum_{r=3}^{k}\left[\prod_{s=r+1}^{k}\left(1-\alpha_{s}\right)\right] \alpha_{r}+\left(1-\alpha_{k}\right) \cdots\left(1-\alpha_{2}\right) \alpha_{1}+\left(1-\alpha_{k}\right) \cdots\left(1-\alpha_{3}\right) \alpha_{2} \\
& \leq \sum_{r=3}^{k}\left[\prod_{s=r+1}^{k}\left(1-\alpha_{s}\right)\right] \alpha_{r}+\left(1-\alpha_{k}\right) \cdots\left(1-\alpha_{3}\right) \quad \text { since } \alpha_{1} \leq 1 \\
& \leq \sum_{r=\ell}^{k}\left[\prod_{s=r+1}^{k}\left(1-\alpha_{s}\right)\right] \alpha_{r}+\left(1-\alpha_{k}\right) \cdots\left(1-\alpha_{\ell}\right) \quad \text { for all } \ell \leq k \\
& \leq 1 .
\end{aligned}
$$

Since

$$
E\left[\prod_{s=r+1}^{k}\left(1-\alpha_{s}\right) \alpha_{r} \eta_{r}(i, j) \mid \mathscr{F}_{r}\right]=\prod_{s=r+1}^{k}\left(1-\alpha_{s}\right) \alpha_{r} E\left[\eta_{r}(i, j) \mid \mathscr{F}_{r}\right]=0,
$$

$\prod_{s=r+1}^{k}\left(1-\alpha_{s}\right) \alpha_{r} \eta_{r}(i, j)$ is a martingale difference. Furthermore, note that

$$
\begin{aligned}
E\left[\left|Y_{k}(i, j)\right|\right] & \leq E\left[\sum_{r=1}^{k}\left[\prod_{s=r+1}^{k}\left(1-\alpha_{s}\right)\right] \alpha_{r}\left|\eta_{r}(i, j)\right|\right] \\
& =\sum_{r=1}^{k}\left[\prod_{s=r+1}^{k}\left(1-\alpha_{s}\right)\right] \alpha_{r} E\left[\left|\eta_{r}(i, j)\right|\right] \\
& =\sum_{r=1}^{k}\left[\prod_{s=r+1}^{k}\left(1-\alpha_{s}\right)\right] \alpha_{r} E\left[E\left[\left|\eta_{r}(i, j)\right| \mid \mathscr{F}_{r}\right]\right] \\
& \leq 2 \sum_{r=1}^{k}\left[\prod_{s=r+1}^{k}\left(1-\alpha_{s}\right)\right] \alpha_{r} \\
& \leq 2 .
\end{aligned}
$$

This shows that $\left\{Y_{k}(i, j)\right\}_{k=1}^{\infty}$ is a $L^{1}$ bounded martingale sequence and remains bounded almost surely by Doob's martingale inequality. Thus, we have from (9) that $\left\{U_{k}(i, j)\right\}_{k=1}^{\infty}$ is bounded w.p.1.

We have the following convergence theorem for MARS-SA.

Theorem 3 Assume the following conditions hold:

(1) $\alpha_{k} \in(0,1) \forall k, \sum_{k=1}^{\infty} \alpha_{k}=\infty$, and $\sum_{k=1}^{\infty} \alpha_{k}^{2}<\infty$;

(2) $T_{k} \geq T_{k+1}>0$ and $T_{k} \rightarrow 0$ as $k \rightarrow \infty$;

(3) $\frac{1}{\alpha_{k}}\left(\frac{1}{T_{k+1}}-\frac{1}{T_{k}}\right) \rightarrow 0$ as $k \rightarrow \infty$.

Then $\hat{q}_{k}(i, j) \rightarrow I\left\{x^{*} \in \Lambda_{i, j}\right\}$ w.p. 1 . as $k \rightarrow \infty$ for all $i, j$.

Theorem 3 implies that the sequence of stochastic matrices $\hat{q}_{k}$ generated at successive iterations of MARS-SA will converge to a limiting matrix that assigns unit mass to the optimal solution $x^{*}$. Condition (1) is a typical stochastic approximation condition; it requires that the gain sequence $\left\{\alpha_{k}\right\}$ should decay to zero at a rate that is neither too fast nor too slow. Condition (2) requires that the annealing schedule $\left\{T_{k}\right\}$ should also decay to zero monotonically. Condition (3) reflects a tradeoff in the choices of $\alpha_{k}$ and $T_{k}$. It stipulates that the annealing of the temperature $T_{k}$ should be sufficiently slow, so that the difference in the inverse temperatures will vanish to zero at rate that is faster than the decay rate of $\alpha_{k}$. Intuitively, since $E\left[\zeta_{k}(i, j) \mid \mathscr{F}_{k}\right]=0,(8)$ is essentially a stochastic approximation procedure for approximating the perpetually iteration-varying summation $\sum_{\mathbb{X}} e^{H(x) / T_{k+1}} I\left\{x \in \Lambda_{i, j}\right\}$, whose varying speed is controlled by the annealing rate of $T_{k}$. Thus, the decay rate in gain $\alpha_{k}$ should be relatively slow in order to allow some proper tracking of the desired quantity as $T_{k}$ decreases to zero. 


\section{Hu and Wang}

Proof of Theorem 3: Dividing both sides of (8) by $\sum_{\mathbb{X}} e^{H(x) / T_{k+1}}$ yields

$$
\begin{aligned}
& U_{k+1}(i, j)=U_{k}(i, j)+\left(1-\alpha_{k}\right) U_{k}(i, j)\left[\frac{\sum_{\mathbb{X}} e^{\frac{H(x)}{T_{k}}}}{\sum_{\mathbb{X}} e^{\frac{H(x)}{T_{k+1}}}}-1\right]+\alpha_{k}\left[\frac{\sum_{\mathbb{X}} e^{\frac{H(x)}{T_{k+1}}} I\left\{x \in \Lambda_{i, j}\right\}}{\sum_{\mathbb{X}} e^{\frac{H(x)}{T_{k+1}}}}-U_{k}(i, j)\right]+\alpha_{k} \eta_{k}(i, j) \\
& =U_{k}(i, j)+\left(1-\alpha_{k}\right) U_{k}(i, j)\left(E_{g_{k+1}}\left[e^{H(X)\left(\frac{1}{T_{k}}-\frac{1}{T_{k+1}}\right)}\right]-1\right)+\alpha_{k}\left(E_{g_{k+1}}\left[I\left\{X \in \Lambda_{i, j}\right\}\right]-U_{k}(i, j)\right)+\alpha_{k} \eta_{k}(i, j) .
\end{aligned}
$$

Define $V_{k}(i, j)=U_{k}(i, j)-I\left\{x^{*} \in \Lambda_{i, j}\right\}$. The above equation can be written in terms of $V_{k}(i, j)$ as

$$
V_{k+1}(i, j)=V_{k}(i, j)-\xi_{k}(i, j)
$$

where

$$
\begin{aligned}
\xi_{k}(i, j)=\alpha_{k} V_{k}(i, j) & +\alpha_{k}\left(I\left\{x^{*} \in \Lambda_{i, j}\right\}-E_{g_{k+1}}\left[I\left\{X \in \Lambda_{i, j}\right\}\right]\right) \\
& +\left(1-\alpha_{k}\right)\left(V_{k}(i, j)+I\left\{x^{*} \in \Lambda_{i, j}\right\}\right)\left(1-E_{g_{k+1}}\left[e^{H(X)\left(\frac{1}{T_{k}}-\frac{1}{T_{k+1}}\right)}\right]\right)-\alpha_{k} \eta_{k}(i, j) .
\end{aligned}
$$

The rest of the proof amounts to showing that $V_{k}(i, j) \rightarrow 0$ w.p.1. as $k \rightarrow \infty$. To this end, we establish that all relevant conditions on the convergence of a time-varying SA recursion in Evans and Weber (1986) are satisfied in our setting.

Let $M_{k}(i, j)=E\left[\xi_{k}(i, j) \mid \mathscr{F}_{k}\right]$ and $Z_{k}(i, j)=\xi_{k}(i, j)-M_{k}(i, j)$.

(i) First, we show that for any $\varepsilon>0, P\left(\left|V_{k}(i, j)\right|>\varepsilon, V_{k}(i, j) M_{k}(i, j)<0\right.$ i.o. $)=0$. We have

$$
\begin{aligned}
V_{k}(i, j) M_{k}(i, j)=\alpha_{k}\left[V_{k}^{2}(i, j)\right. & +V_{k}(i, j)\left(I\left\{x^{*} \in \Lambda_{i, j}\right\}-E_{g_{k+1}}\left[I\left\{X \in \Lambda_{i, j}\right\}\right]\right) \\
& \left.+\frac{1-\alpha_{k}}{\alpha_{k}} V_{k}(i, j)\left(V_{k}(i, j)+I\left\{x^{*} \in \Lambda_{i, j}\right\}\right)\left(1-E_{g_{k+1}}\left[e^{H(X)\left(\frac{1}{T_{k}}-\frac{1}{T_{k+1}}\right)}\right]\right)\right] .
\end{aligned}
$$

By Lemma 2, $V_{k}(i, j)$ is bounded w.p.1. This, together with Lemma 1, shows that the second term in the square brackets above vanishes to zero w.p.1. as $k \rightarrow \infty$. Furthermore, note that

$$
\begin{aligned}
0 & \leq 1-E_{g_{k+1}}\left[e^{H(X)\left(\frac{1}{T_{k}}-\frac{1}{T_{k+1}}\right)}\right] \\
& \leq 1-e^{E_{g_{k+1}}[H(X)]\left(\frac{1}{T_{k}}-\frac{1}{T_{k+1}}\right)} \text { by Jensen's inequality } \\
& \leq 1-e^{-H\left(x^{*}\right)\left(\frac{1}{T_{k+1}}-\frac{1}{T_{k}}\right)} \\
& \leq e H\left(x^{*}\right)\left(\frac{1}{T_{k+1}}-\frac{1}{T_{k}}\right) \text { for } k \text { sufficiently large, }
\end{aligned}
$$

where the last inequality follows from the fact that $e^{-a} \geq 1-e \cdot a$ for $a \in[0,1]$, since we can make $H\left(x^{*}\right)\left(\frac{1}{T_{k+1}}-\frac{1}{T_{k}}\right) \leq 1$ by choosing a value of $k$ that is sufficiently large. Consequently, condition (3) in Theorem 3 implies that the third term in the square brackets also vanishes to zero as $k \rightarrow \infty$. Therefore, w.p.1. we must have $V_{k}(i, j) M_{k}(i, j)>0$ whenever $\left|V_{k}(i, j)\right|>\varepsilon$, i.e., $P\left(\left|V_{k}(i, j)\right|>\varepsilon, V_{k}(i, j) M_{k}(i, j)<0\right.$ i.o. $)=0$.

(ii) Since $V_{k}(i, j)$ is bounded w.p.1. by Lemma 2 and $\alpha_{k} \rightarrow 0$ by condition (1), we must have $\left|M_{k}(i, j)\right|(1+$ $\left|V_{k}(i, j)\right|^{-1} \rightarrow 0$ as $k \rightarrow \infty$ w.p.1. This shows condition (ii) in Evans and Weber (1986).

(iii) $Z_{k}(i, j)=\xi_{k}(i, j)-M_{k}(i, j)=-\alpha_{k} \eta_{k}(i, j)$. Thus,

$$
\sum_{k=1}^{\infty} E\left[\left|Z_{k}(i, j)\right|^{2}\right]=\sum_{k=1}^{\infty} \alpha_{k}^{2} E\left[E\left[\eta_{k}^{2}(i, j) \mid \mathscr{F}_{k}\right]\right]
$$




$$
\begin{aligned}
& =\sum_{k=1}^{\infty} \alpha_{k}^{2} \frac{1}{N} \frac{1}{\left(\sum_{\mathbb{X}} e^{\frac{H(x)}{T_{k+1}}}\right)^{2}}\left\{E\left[E\left[e^{\frac{2 H(X)}{T_{k+1}}} \hat{\phi}^{-2}\left(X, \hat{q}_{k}\right) I\left\{X \in \Lambda_{i, j}\right\} \mid \mathscr{F}_{k}\right]\right]-\left(\sum_{\mathbb{X}} e^{\frac{H(x)}{T_{k+1}}} I\left\{X \in \Lambda_{i, j}\right\}\right)^{2}\right\} \\
& =\sum_{k=1}^{\infty} \alpha_{k}^{2} \frac{1}{N}\left\{E\left[E_{g_{k+1}}\left[\frac{g_{k+1}(X)}{\hat{\phi}\left(X, \hat{q}_{k}\right)} I\left\{X \in \Lambda_{i, j}\right\} \mid \mathscr{F}_{k}\right]\right]-E_{g_{k+1}}^{2}\left[I\left\{X \in \Lambda_{i, j}\right\}\right]\right\} .
\end{aligned}
$$

Since $g_{k+1}(x) \leq 1$ and $\hat{\phi}\left(x, \hat{q}_{k}\right) \geq \beta(1 / m)^{n}$ for all $x \in \mathbb{X}$, there exists a positive constant $C$ such that

$$
\sum_{k=1}^{\infty} E\left[\left|Z_{k}(i, j)\right|^{2}\right] \leq \frac{C}{N} \sum_{k=1}^{\infty} \alpha_{k}^{2}<\infty
$$

by condition (1) in Theorem 3 .

(iv) Finally, we show that $P\left(\liminf _{k \rightarrow \infty}\left|V_{k}(i, j)\right|>0, \sum_{k=1}^{\infty}\left|M_{k}(i, j)\right|<\infty\right)=0$. Note that

$$
\begin{aligned}
\left|M_{k}(i, j)\right| \geq \alpha_{k}\left[\left|V_{k}(i, j)\right|\right. & -\left|I\left\{x^{*} \in \Lambda_{i, j}\right\}-E_{g_{k+1}}\left[I\left\{X \in \Lambda_{i, j}\right\}\right]\right| \\
& \left.-\frac{1-\alpha_{k}}{\alpha_{k}}\left(\left|V_{k}(i, j)\right|+I\left\{x^{*} \in \Lambda_{i, j}\right\}\right)\left(1-E_{g_{k+1}}\left[e^{H(X)\left(\frac{1}{T_{k}}-\frac{1}{T_{k+1}}\right)}\right]\right)\right] .
\end{aligned}
$$

Let $\Omega_{1}=\left\{\liminf _{k \rightarrow \infty}\left|V_{k}(i, j)\right|>0\right\}, \Omega_{2}=\left\{\sum_{k=1}^{\infty}\left|M_{k}(i, j)\right|<\infty\right\}$, and $\Omega_{3}=\left\{V_{k}(i, j)\right.$ is bounded $\}$. For each $\omega \in \Omega_{1}$, there exists a $\delta(\omega)$ such that $\liminf _{k \rightarrow \infty}\left|V_{k}(i, j)\right|>\delta(\omega)>0$. Therefore, we can find a $K_{\delta}(\omega)>0$ such that $\left|V_{k}(i, j)\right| \geq \delta(\omega)$ for all $k \geq K_{\delta}(\omega)$. On the other hand, for each $\omega \in \Omega_{3}$, we can find a $K_{\delta}^{\prime}(\omega)>0$ such that for all $k \geq K_{\delta}^{\prime}(\omega)$,

$\left|I\left\{x^{*} \in \Lambda_{i, j}\right\}-E_{g_{k+1}}\left[I\left\{X \in \Lambda_{i, j}\right\}\right]\right|+\frac{1-\alpha_{k}}{\alpha_{k}}\left(\left|V_{k}(i, j)\right|+I\left\{x^{*} \in \Lambda_{i, j}\right\}\right)\left(1-E_{g_{k+1}}\left[e^{H(X)\left(\frac{1}{T_{k}}-\frac{1}{T_{k+1}}\right)}\right]\right) \leq \delta(\omega) / 2$.

Thus, for each $\omega \in \Omega_{1} \cap \Omega_{3}$, we have $\left|M_{k}(i, j)\right|>\alpha_{k} \delta(\omega) / 2$ whenever $k \geq K_{\delta}^{*}(\omega):=\max \left\{K_{\delta}(\omega), K_{\delta}^{\prime}(\omega)\right\}$. It follows that

$$
\sum_{k=1}^{\infty}\left|M_{k}\right|>\frac{\delta(\omega)}{2} \sum_{k=K_{\delta}^{*}(\omega)}^{\infty} \alpha_{k}=\infty \text { by condition (1). }
$$

This implies $P\left(\Omega_{1} \cap \Omega_{2} \cap \Omega_{3}\right)=0$. Finally, $P\left(\Omega_{1} \cap \Omega_{2}\right)=P\left(\Omega_{1} \cap \Omega_{2} \cap \Omega_{3}\right)+P\left(\Omega_{1} \cap \Omega_{2} \cap \Omega_{3}^{c}\right) \leq P\left(\Omega_{3}^{c}\right)=0$ by Lemma 2 .

Thus, by directly Theorem 2.2 in Evans and Weber (1986) gives $V_{k}(i, j) \rightarrow 0$ w.p.1. as $k \rightarrow \infty$, i.e., $\lim _{k \rightarrow \infty} U_{k}(i, j)=I\left\{x^{*} \in \Lambda_{i, j}\right\}$ w.p.1. This further shows that

$\lim _{k \rightarrow \infty} \hat{q}_{k}(i, j)=\lim _{k \rightarrow \infty} \frac{S_{k}(i, j) / \sum_{\mathbb{X}} e^{H(x) / T_{k}}}{\sum_{j=1}^{m} S_{k}(i, j) / \sum_{\mathbb{X}} e^{H(x) / T_{k}}}=\lim _{k \rightarrow \infty} \frac{U_{k}(i, j)}{\sum_{j=1}^{m} U_{k}(i, j)}=\frac{I\left\{x^{*} \in \Lambda_{i, j}\right\}}{\sum_{j=1}^{m} I\left\{x^{*} \in \Lambda_{i, j}\right\}}=I\left\{x^{*} \in \Lambda_{i, j}\right\}$ w.p.1.

\section{NUMERICAL RESULTS}

In this section, we illustrate effectiveness of the proposed MARS-SA algorithm on several benchmark problems and compare its performance with that of the MARS algorithm of $\mathrm{Hu}$ and $\mathrm{Hu}$ (2010).

We consider the following four discrete optimization problems.

$$
\begin{gathered}
\text { Discrete Weighted-Sphere function }\left(n=15, x_{i} \in\{-5+0.5 \ell, \ell=0, \ldots, 20\}, i=1, \ldots, n\right) \\
\qquad H_{1}(x)=-\sum_{i=1}^{n} i x_{i}^{2},
\end{gathered}
$$

where $x^{*}=(0, \ldots, 0)^{T}$ and $H_{1}\left(x^{*}\right)=0$. 
(2)

Discrete Rastrigin function $\left(n=15, x_{i} \in\{-5+0.5 \ell, \ell=0, \ldots, 20\}, i=1, \ldots, n\right)$

$$
H_{2}(x)=-\sum_{i=1}^{n}\left(x_{i}^{2}-10 \cos \left(2 \pi x_{i}\right)\right)-10 n
$$

where $x^{*}=(0, \ldots, 0)^{T}$ and $H_{2}\left(x^{*}\right)=0$.

Discrete Griewank function $\left(n=15, x_{i} \in\{-5+0.5 \ell, \ell=0, \ldots, 20\}, i=1, \ldots, n\right)$

$$
H_{3}(x)=-\frac{1}{40} \sum_{i=1}^{n} x_{i}^{2}+100 \prod_{i=1}^{n} \cos \left(\frac{x_{i}}{\sqrt{i}}\right)-100,
$$

where $x^{*}=(0, \ldots, 0)^{T}, H_{3}\left(x^{*}\right)=0$.

Trigonometric function $\left(n=15, x_{i} \in\{-5+0.5 \ell, \ell=0, \ldots, 20\}, i=1, \ldots, n\right)$

$$
H_{4}(x)=-1-\sum_{i=1}^{n}\left[8 \sin ^{2}\left(7\left(x_{i}-0.9\right)^{2}\right)+6 \sin ^{2}\left(14\left(x_{i}-0.9\right)^{2}\right)+\left(x_{i}-0.9\right)^{2}\right],
$$

where $x^{*}=(1, \ldots, 1)^{T}, H_{4}\left(x^{*}\right)=-3.4895$.

Since all objective functions above are negative valued, whereas $H(x)$ is assumed to be non-negative in our analysis, we have used an additional increasing performance function $S(x)=80+\frac{x}{1+0.0125|x|}$, and for each of the four test cases, we maximize $S(H(x))$ instead of directly maximizing $H(x)$. The following set of parameters are used in MARS-SA: a relatively conservative step-size/gain $\alpha_{k}=2 /(k+100)^{0.501}$ (cf. e.g., Spall 2003), a Cauchy annealing type temperature schedule $T_{k}=1 / \sum_{t=1}^{k} \frac{1}{t}$ (Dukkipati, Narasimha, and Bhatnagar 2004), a constant sample size $N=2$, and a mixing parameter $\beta=0.1$. Note that the above parameter setting satisfies the conditions in Theorem 3 for convergence. The same set of parameter values are used in implementing the original MARS algorithm, except that we have considered two different sample sizes: a constant sample size $N=2$ and a polynomially increasing (iteration-dependent) sample size $N_{k}=\max \left\{2,\left\lfloor k^{0.502}\right\rfloor\right\}$, where $\lfloor a\rfloor$ is the largest integer no greater than $a$.

For each test case, we performed 50 independent replication runs of both algorithms. The comparison results are summarized in Figure 1, which plots the averaged current best objective function values as a function of the number of performance evaluations consumed thus far. The figure clearly indicates the convergence of MARS-SA and the MARS algorithm with a polynomial sample size per iteration, with MARS-SA providing superior performance over the original MARS. Moreover, for test functions $H_{1}, H_{2}$, and $H_{4}$, we see that MARS-SA consistently finds global optimal solutions in all runs even when the per-iteration sample size is set to $N=2$, whereas the original MARS algorithm with $N=2$ does not seem to converge to the correct global optima.

\section{CONCLUSIONS}

In this paper, we have presented a new algorithm for solving discrete black-box optimization problems. The algorithm can essentially be viewed as a variant of the recently proposed MARS algorithm for global optimization, but improves MARS with an additional stochastic averaging scheme. Such a stochastic averaging procedure makes a more efficient use of the past sampling information, and eliminates the polynomially increasing (per-iteration) computational requirement of MARS in constructing surrogate distributions. Under mild regularity conditions, we have shown that the algorithm converges to the global optimal solution even when the number of samples per iteration is fixed at a small constant value. Our preliminary numerical results suggest that the proposed algorithm could lead to improved performance over the original MARS method. 

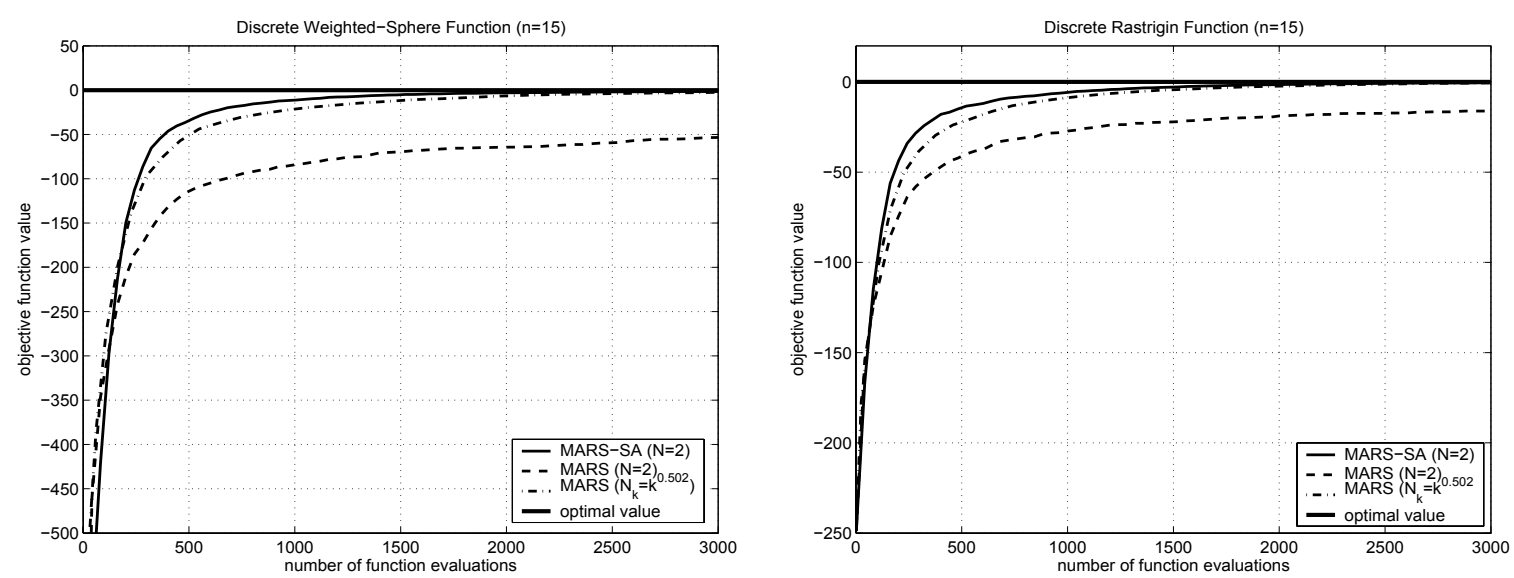

$H_{1}$

$\mathrm{H}_{2}$

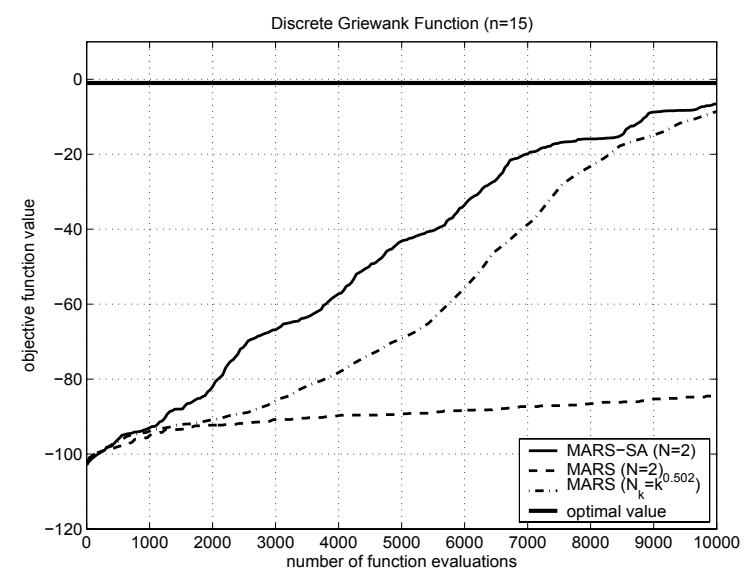

$\mathrm{H}_{3}$

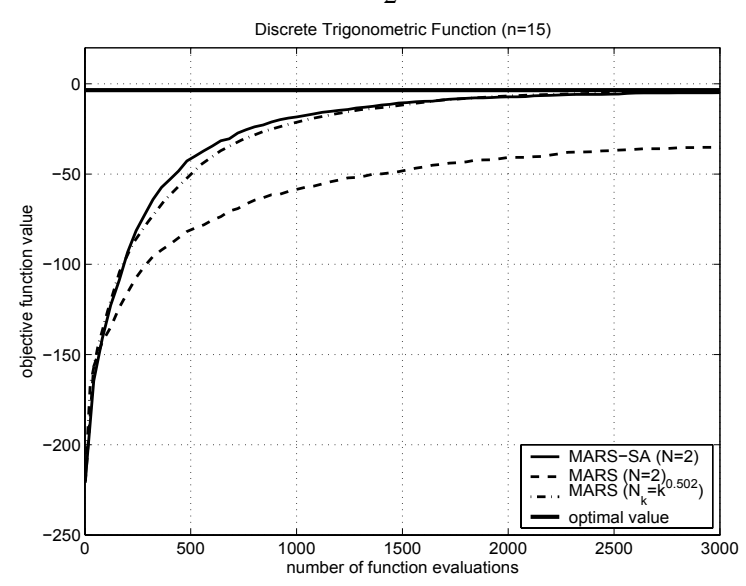

$H_{4}$

Figure 1: Average Performance of MARS-SA vs. MARS on test functions $H_{1}-H_{4}$.

\section{ACKNOWLEDGMENTS}

This work was supported in part by the Air Force Office of Scientific Research under Grant FA95501010340, and by the National Science Foundation under Grants CMMI-0900332 and CMMI-1130761.

\section{REFERENCES}

Dorigo, M., and L. M. Gambardella. 1997. "Ant colony system: A cooperative learning approach to the traveling salesman problem". IEEE Transactions on Evolutionary Computation 1:53-66.

Dukkipati, A., M. Narasimha, and S. Bhatnagar. 2004. "Cauchy annealing schedule: an annealing schedule for Boltzmann selection scheme in evolutionary algorithms". In Proceedings of the Congress on Evolutionary Computation, 55-62.

Evans, S. N., and N. C. Weber. 1986. "On the Almost Sure Convergence of A General Stochastic Approximation Procedure". Bulletin of the Australian Mathematical Society 34:335-342.

Glover, F. W. 1990. "Tabu search: a tutorial”. Interfaces 20:74-94.

Goldberg, D. E. 1989. Genetic algorithms in search, optimization, and machine learning. Boston, MA: Academic Publishers.

Hu, J., M. C. Fu, and S. I. Marcus. 2007. "A model reference adaptive search algorithm for global optimization". Operations Research 55:549-568. 
$\mathrm{Hu}$, J., and P. Hu. 2009, December. "On the performance of the cross-entropy method". In Proceedings of the 2009 Winter Simulation Conference, edited by M. D. Rossetti, R. R. Hill, B. Johansson, A. Dunkin, and R. G. Ingalls, 459-468. Piscataway, New Jersey: Institute of Electrical and Electronics Engineers, Inc.: Institute of Electrical and Electronics Engineers, Inc.

$\mathrm{Hu}$, J., and P. Hu. 2010, December. "An approximate annealing search algorithm to global optimization and its connections to stochastic approximation". In Proceedings of the 2010 Winter Simulation Conference, edited by B. Johansson, S. Jain, J. Montoya-Torres, J. Hugan, and E. Yücesan, 1223-1234. Piscataway, New Jersey: Institute of Electrical and Electronics Engineers, Inc.: Institute of Electrical and Electronics Engineers, Inc.

Kirkpatrick, S., C. D. Gelatt, and M. P. Vecchi. 1983. "Optimization by simulated annealing”. Science 220:671-680.

Kushner, H. J., and G. G. Yin. 1997. Stochastic approximation algorithms and applications. New York, NY: Springer-Verlag.

Larranaga, P., and J. A. Lozano. 2002. Estimation of distribution algorithms: a new tool for evolutionary computation. Boston, MA: Kluwer Academic Publishers.

Robbins, H., and S. Monro. 1951. "A stochastic approximation method". Annals of Mathematical Statistics 22:400-407.

Romeijn, H. E., and R. L. Smith. 1994. "Simulated annealing and adaptive search in global optimization". Probability in the Engineering and Informational Sciences 8:571-590.

Rubinstein, R. Y., and D. P. Kroese. 2004. The cross-entropy method: A unified approach to combinatorial optimization, Monte-Carlo simulation, and machine learning. New York, NY: Springer.

Shi, L., and S. Ólafsson. 2000. "Nested partitions method for global optimization". Operations Research 48:390-407.

Spall, J. C. 2003. Introduction to stochastic search and optimization. Hoboken, NJ: John Wiley \& Sons.

Wolpert, D. H. 2004. "Finding Bounded Rational Equilibria Part I: Iterative Focusing". In Proceedings of the International Society of Dynamic Games Conference, edited by T. Vincent.

Zabinksy, Z. B. 2003. Stochastic adaptive search for global optimization. Norwell, MA: Kluwer Academic Publishers.

\section{AUTHOR BIOGRAPHIES}

JIAQIAO HU is an Assistant Professor in the Department of Applied Mathematics and Statistics at the State University of New York, Stony Brook. He received a B.S. in automation from Shanghai Jiao Tong University, an M.S. in applied mathematics from the University of Maryland, Baltimore County, and a $\mathrm{Ph} . \mathrm{D}$. in electrical engineering from the University of Maryland, College Park. His research interests include Markov decision processes, applied probability, and simulation optimization. His e-mail address is jqhu@ams.sunysb.edu.

CHEN WANG is a Ph.D. Student in the Department of Applied Mathematics and Statistics at the State University of New York, Stony Brook. She received the B.S. degree in statistics from Fudan University, Shanghai, China, in 2010. Her research interests are simulation optimization, mathematical modeling, and statistical analysis. Her e-mail address is chenwang@ams.sunysb.edu. 